\title{
Stratégies de résistance des agriculteurs familiaux face à l'avancée de l'agriculture capitaliste en Uruguay
}

\author{
Virginia Rossi ${ }^{1, *}$, Verónica Filardo ${ }^{2}$ et Eduardo Chia ${ }^{3}$ \\ ${ }^{1}$ Departamento de Ciencias Sociales, Facultad de Agronomía, Universidad de la República; Ruta 3 km 363, EEMAC, 60.000, Paysandú, \\ Uruguay \\ ${ }^{2}$ Departamento de Sociología, Facultad de Ciencias Sociales, Universidad de la República, Montevideo, Uruguay \\ ${ }^{3}$ UMR Innovation, INRA, Montpellier, France
}

\begin{abstract}
Résumé - La résistance des agriculteurs familiaux à l'avancée de l'agriculture capitaliste se traduit par des pratiques et des stratégies économiques, sociales et techniques singulières, qui leur confèrent des capacités de reproduction spécifiques. Notre principale hypothèse est la suivante : les modèles d'action à l'origine de la résistance des agriculteurs familiaux dépendent du contexte, de leur situation, de leurs projets et des valeurs qu'ils associent au métier d'agriculteur. Ces modèles sont situés historiquement et fabriqués collectivement. Nous avons développé une approche «compréhensive » des éleveurs familiaux en Uruguay et mobilisé le concept d'habitus afin d'identifier et d'étudier leurs pratiques et stratégies de résistance. Á partir des macro-pratiques économiques, symboliques, éducatives et successorales nous avons identifié trois types de résistance : «Rendre la famille autonome», « Transmettre le patrimoine familial » et «Rester dans le réseau territorial ». L'habitus permet aux éleveurs étudiés de fabriquer et de maintenir une logique familiale «paysanne» à la base de leur stratégie de résistance.
\end{abstract}

Mots clés : anthropologie sociale / agriculture familiale / moyens de survie / habitus paysan / Uruguay

\begin{abstract}
Resistance strategies of family farmers facing agribusiness extension in Uruguay. The resistance of family farmers facing agribusiness extension is reflected in unique economic, social and technical practices and strategies, which give them specific reproductive capacities. Our main hypothesis is that the action models behind the resistance of family farmers depend on the context of their situations, their projects and the values they associate with the farming profession and are historically located and collectively produced. We have developed a comprehensive approach and mobilized the concept of habitus to identify and study the practices and strategies of resistance. From economic, symbolic, educational and inheritance macro-practices (fields) we have identified three types of resistance: "Preserve the autonomy of the peasant family", "Transmitting the family legacy", "Staying in the social network". The habitus allows family farmers to build and maintain a "peasant" family logic and define the "profession" of the farmer. This last dimension remains to be further explored.
\end{abstract}

Keywords: social anthropology / family farming / livelihoods / peasant habitus / Uruguay

\section{Introduction}

En Uruguay, l'élevage familial, malgré les nombreuses crises qui l'ont frappé, a su évoluer tout en conservant les conditions de vie rurale ainsi que ses valeurs (Rossi, 2017). Cependant, comme dans la plupart des pays latino-américains, le processus d'appropriation des terres («land grabbing»), accéléré depuis les années 1980, affecte la production familiale et entrepreneuriale (Piñeiro, 2014), ce qui élargit le fossé qui

*Auteur de correspondance : virossi@fagro.edu.uy sépare petites et grandes exploitations. En 2014, les exploitations de moins de 100 hectares représentaient $56 \%$ du total des exploitations et disposaient de moins de $5 \%$ de la surface agricole utile (SAU), tandis que celles de plus de 1000 ha (9\% du total) disposaient de plus de $60 \%$ de la SAU (MGAP, 2014). Ce sont les plus petites exploitations (possédant moins de 20 hectares) qui disparaissent : en effet $67 \%$ des 12000 exploitations uruguayennes existant auparavant ont disparu entre 2000 et 2011 (Carámbula, 2015). Oyhantçabal et Narbondo (2019) montrent que, depuis les années 2000, plus de $48 \%$ de la SAU de l'Uruguay (estimée à 16,3 millions d'hectares) a été vendue, malgré l'augmentation du prix des 
terres agricoles entre 2000 et 2011 (multiplié par 7); le nombre de propriétaires uruguayens a diminué, passant de $90 \%$ à $54 \%$ du total des exploitations agricoles, au profit des sociétés anonymes. On constate, pour la même période, d'une part une expansion de la sylviculture (monoculture d'eucalyptus), dont les terres sont passées de 4000 à 1 million d'hectares et, d'autre part, de l'agriculture de «rente»-principalement le soja (MGAP, 2018).

Néanmoins, l'agriculture familiale reste importante en Uruguay: elle représente 36965 producteurs travaillant dans 21657 exploitations agricoles (48\% des 44781 exploitations que compte le pays) où la production bovine et ovine représente la principale source de revenus (MGAP, 2018). Mais l'avancée de l'agro-industrie génère une situation conflictuelle entre les tenants de l'agriculture familiale (petits producteurs) et ceux de l'agriculture capitaliste (grands propriétaires) (Achkar et al., 2006). Ce n'est que depuis 2009 que l'agriculture familiale bénéficie d'un soutien de la part de l'État par le biais de politiques publiques d'appui spécifique. Malgré cet appui tardif, le soutien à l'agriculture familiale en Uruguay est devenu une préoccupation nationale, comme en témoigne la loi sur l'agro-écologie votée par le parlement en décembre 2018 (Ley No. 19717, «Plan Nacional para el fomento de la producción con bases agroecológicas»), dont la principale cible est les agriculteurs familiaux et qui vise le renforcement de la sécurité alimentaire et la protection des ressources naturelles.

Plusieurs ouvrages (Piñeiro, 1985; Chia et Dugué, 2006; Schneider, 2016; Rossi, 2017) exposent l'importance des capacités d'innovation, des stratégies et des pratiques de "résistance» des agriculteurs familiaux leur permettant d'améliorer leur fonctionnement et de continuer à exercer le métier d'agriculteur. Certains auteurs s'interrogent sur la pertinence du concept d'agriculture familiale pour comprendre et élaborer des politiques publiques (Chia, 1990; Ploeg, 2010; Gasselin et al., 2014; Sourisseau, 2014). Selon Schneider (2016), il faut privilégier une connaissance précise des stratégies et des pratiques de production des agriculteurs familiaux pour pouvoir élaborer de bonnes politiques publiques de développement rural. Cependant, les mécanismes et les modes de résistance développés par les agriculteurs familiaux sont peu étudiés. Nous proposons de contribuer à combler ce manque de connaissances, en prenant l'exemple de l'Uruguay.

Nous présenterons tout d'abord la notion d'habitus proposée par Bourdieu, puis nous décrirons la méthodologie « compréhensive » que nous avons utilisée afin d'identifier les pratiques et stratégies de résistance des éleveurs familiaux situés dans quatre localités du nord-est de l'Uruguay. Notre hypothèse est que les modèles d'action des producteurs à l'origine de leur résistance dépendent du contexte dans lequel ils se trouvent (leur situation et leurs projets) et des valeurs qu'ils associent au métier d'agriculteur. Nous présenterons ensuite les stratégies de résistance d'un panel d'éleveurs familiaux uruguayens. Nous conclurons en présentant une grille construite selon deux axes: le niveau d'action et le registre d'action, afin de comprendre comment les pratiques s'articulent pour donner naissance à des formes de résistance.

\section{L’habitus pour résister}

La résistance à l'avancée capitaliste dans l'agriculture doit être située et contextualisée car elle peut prendre de nombreuses formes. En Uruguay, Piñeiro (1985) explique que la reproduction sociale se traduit par la résistance individuelle et collective à l'appropriation des excédents, avec des stratégies fondées sur l'économie. Ploeg (2010) relève trois aspects qu'il considère fondamentaux concernant la résistance des agriculteurs à la marchandisation : premièrement, la construction de l'autonomie permettant de résister à la subordination, la dépendance et la privation; deuxièmement, la création, la reproduction et le développement d'une base de ressources autocontrôlées, qui permet la coproduction; et enfin, les multiples interactions avec des marchés diversifiés afin de garantir les revenus et faciliter la reproduction de la base de ressources.

\subsection{Habitus, domaines de pouvoir, sens pratique}

D'après Bourdieu (1991), les habitus sont le produit de l'intériorisation des structures objectives (assimilation des comportements) qu'on acquiert à travers une expérience durable dans une organisation sociale dans laquelle on s'inscrit. Il s'agit des dispositifs d'actions, de pensées, de perceptions nous faisant agir d'une certaine façon plutôt que d'une autre et opérant de manière inconsciente («constructivisme structuraliste »). Nous pouvons les comprendre en tant que principes générateurs de styles de vie, retraduisant les caractéristiques inhérentes et relationnelles d'une position sociale commune à un groupe de personnes (Bourdieu, 2001).

La notion de « domaine de pouvoir » est également utilisée pour comprendre l'action stratégique des groupes sociaux, qui mobilisent différents types de capitaux (pas seulement le capital économique) afin d'améliorer ou de conserver des positions hiérarchiques, des groupes sociaux, à l'intérieur de l'espace social (Bourdieu, 2001). L'action et la mobilisation des ressources matérielles et symboliques sont déterminées par l'intérêt (illusio) des agents à se maintenir dans la situation. Utilisant la métaphore du jeu, Bourdieu et Wacquant (2008) expliquent que l'intérêt se traduit par «un investissement » permettant de «rester dans le jeu» et de croire «qu'il vaut la peine d'être joué ». De manière dialectique, nous pouvons aujourd'hui représenter, d'une part, l'intérêt de dominer ou de conserver des positions déjà acquises par les agents des entreprises agroalimentaires; et, d'autre part, la résistance à cette domination des agriculteurs familiaux organisés au sein de la CNFR (Comisión Nacional de Fomento Rural, réunissant plus de 17000 agriculteurs, représentés par 100 syndicats et associations). Pour finir, la notion de «sens pratique» fait allusion à l'existence d'une raison inhérente aux pratiques, allant plus loin que la maximisation du bénéfice économique et que Bourdieu appelle «une économie des pratiques». Ainsi, dans la plupart des conduites ordinaires les agents agissent au moyen de schémas codifiés, qui réduisent le plus possible l'équivoque et l'imprécision, en particulier dans les interactions. Il s'agit d'une logique pratique («modus operandi») déterminant des actions raisonnables et adaptées à leur but sans être le produit d'un projet ou d'un plan (Bourdieu, 1991 : 89).

\subsection{Pratiques de résistance et habitus paysan}

La résistance peut prendre différentes formes. Scott (1989) souligne l'importance de la «résistance quotidienne»: une 
forme d'action politique « informelle, tacite et déguisée » menée par des groupes subordonnés. Elle se caractérise par des actes tels que la dissimulation, la feinte, la sortie (exit) ou le sabotage. Selon Reed-Danahay (2007), cette définition est très proche de la notion de « pratiques quotidiennes » proposée par De Certeau (1980), de celle de «bricolage» développé par Levi-Strauss (1962), ou encore de celle d' « hybridation » de Latour(1993). En ce sens, les pratiques quotidiennes associées à la reproduction sociale des exploitations familiales, sans qu'elles soient des actes conscients de résistance, peuvent être comprises comme faisant partie d'une stratégie (avec des finalités), qui se transmet socialement au travers de l'habitus du paysan. Cette notion d'habitus comme «subjectivité socialisée» (Bourdieu, 2001) suppose de considérer le paysan comme un «individu collectif» et permet de mettre en relation les principes et les valeurs qui fabriquent son style de vie avec un ensemble de pratiques et de stratégies qui en sont la base. Selon différentes perspectives et espaces sociaux, les auteurs font référence à des formes de résistance à la domination, « cachées » dans les pratiques quotidiennes ou dans les discours des subordonnés, qui fonctionnent comme des mécanismes de résistance efficaces même dans les conditions de domination auxquelles ils sont soumis. Afin de comprendre l'action stratégique des producteurs familiaux, nous utilisons la notion d' « habitus paysan » qui se réfère à leur habitus en tant que groupe social. Cela signifie qu'il existe des pratiques symboliques qui déterminent une certaine autonomie éthique en rapport à l'économie de marché et qu'en conséquence, pour comprendre les pratiques des agriculteurs familiaux, il est nécessaire de mettre en jeu un certain ordre moral (Bourdieu, 2001).

De façon complémentaire, on considère l'existence d'un système stratégique de reproduction sociale des exploitations familiales permettant le développement de pratiques que l'on appellera «pratiques de résistance». Pour Bourdieu (1994), les stratégies de reproduction, au nombre de quatre (successorales, éducatives, d'investissement économique et d'investissement symbolique), constituent un système de reproduction. Bien qu'elles soient toutes entremêlées et interdépendantes, les stratégies s'articulent chronologiquement au fur et à mesure que les priorités changent au cours du cycle familial (Bourdieu, 1994).

Les pratiques et stratégies des résistances sont le produit des actions individuelles et collectives. En ce sens, il nous paraît intéressant de mobiliser les travaux de Bourdieu (2001) afin de comprendre la façon dont sont agencées les pratiques individuelles et collectives. Le sens pratique et l'action stratégique ne sont pas le produit d'une intention consciente et rationnelle selon Bourdieu (1991: 89). Ce sont les dispositions d'un habitus qui tendent spontanément à reproduire les conditions de sa propre production. Même si la notion de stratégie, en tant que produit d'un sens pratique, se trouve historiquement définie (acquise depuis les jeux d'enfants), elle suppose aussi une invention (innovation) permanente, indispensable pour s'adapter à des situations variées, jamais parfaitement identiques : c'est l'art d'innover.

\section{Comment accéder aux pratiques des agriculteurs?}

Afin d'identifier les stratégies et pratiques de résistance des éleveurs, nous avons privilégié une approche qualitative
(Dumez, 2011) auprès des éleveurs familiaux. L'objet analysé est l'exploitation agricole dans toute sa globalité et sa complexité, ce qui inclut la famille. La méthodologie se structure suivant trois postulats: i) il n'est pas possible de comprendre séparément les unités de production et la famille : c'est un « système famille-exploitation » (Osty, 1978); ii) il y a un cadre de «raisonnabilité » dans les pratiques des producteurs, une «logique pratique» (Bourdieu, 1991); iii) un système de résistance peut réunir différents types de stratégies selon les contextes, les trajectoires et le cycle familial (Bourdieu, 1994).

Cette recherche a été réalisée dans le cadre d'un accord de coopération entre la CNFR et la station expérimentale EEMAC (Estación Experimental «Dr. Mario A. Cassinoni») de l'Universidad de la República, Paysandú, Uruguay. L'objectif était de comprendre les stratégies de résistance des éleveurs familiaux face à l'avancée de l'agrobusiness. Dans l'optique de choisir quels cas nous allions étudier, nous avons tenu compte de la grande diversité des éleveurs dans la zone d'étude et de leur appartenance et ancienneté au sein des syndicats.

Notre échantillon est composé de 16 exploitations familiales situées dans 4 localités différentes: nous avons donc 4 cas par zone (Fig. 1). Dans les entretiens, nous avons caractérisé la trajectoire socio-productive des familles (état civil, nombre d'enfants, nombre d'années d'ancienneté en tant que producteur...) et la présence du travail familial, le système de production ainsi que les pratiques productives (production, commercialisation, investissements, prélèvements privés, etc.) (Tab. 1). Les contextes de recherche numérotés 1 et 2 correspondent à des régions d'élevage du Basalto, avec une prédominance de pâturages naturels, d'un élevage mixte de bovins/ovins et différents niveaux d'extensivité. Ils sont situés dans des localités où les conditions de sols ainsi que la capacité de stockage de l'eau du sol ne permettent pas la mise en place de systèmes de production plus intensifs, interdisant l'engraissement du bétail (hivernage). Les contextes 3 et 4 sont situés dans la zone côtière du fleuve Uruguay, une région plus diversifiée qui comprend de petites villes, où se développent des systèmes d'agriculture familiale de différents niveaux d'intensification.

Nous avons utilisé la méthode «Approche globale des exploitations agricoles »(AGEA - ; Marshall et al., 1994) pour étudier les pratiques productives des agriculteurs. Cette méthode combine données qualitatives et données quantitatives et permet d'étudier le fonctionnement des exploitations agricoles. Elle comporte 4 étapes: Étape 1, Histoire de l'exploitation et de la famille, identification des objectifs et finalités ; Étape 2, Diagnostic du système de production, des stratégies d'investissement et des pratiques productives; Étape 3, Élaboration du schéma de fonctionnement ; Étape 4, restitution, validation et amélioration du diagnostic qui se déroulent à intervalles réguliers (1 mois dans notre cas) et permettent ainsi d'établir des relations de confiance avec les agriculteurs. Les entretiens ont été réalisés entre 2012 et 2013. Au début de chaque entretien, une restitution des données antérieures scrupuleusement analysées est faite à l'agriculteur et à sa famille. Cela permet aux agriculteurs, non seulement d'avoir un retour, mais aussi de vérifier et valider les analyses réalisées. Une réunion collective a été organisée avec les 16 producteurs afin de valider les schémas de 


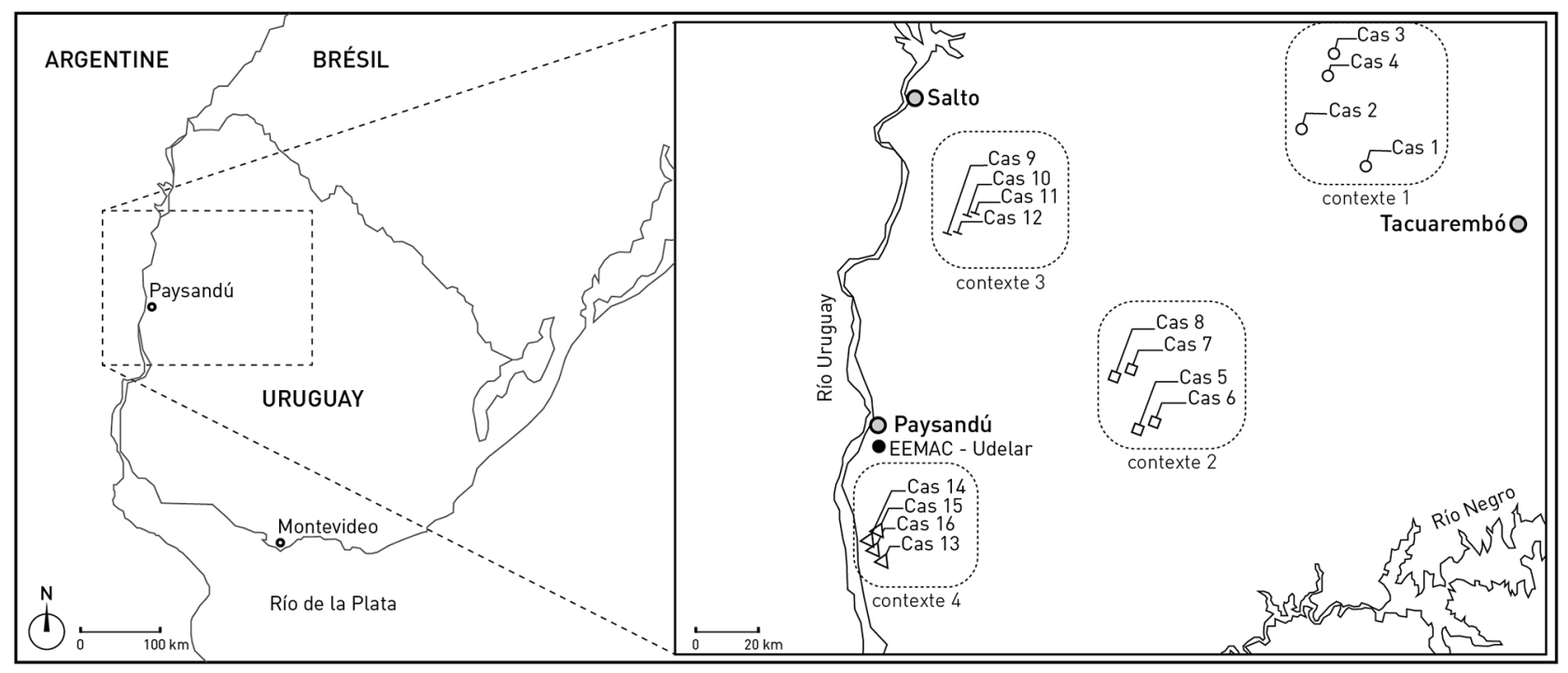

Fig. 1. Localisation des territoires et des cas étudiés.

Fig. 1. Location of territories and case studies.

Tableau 1. Principales caractéristiques des exploitations étudiées.

Table 1. Main characteristics of the case studies.

\begin{tabular}{|c|c|c|c|c|}
\hline Contextes de recherche & Cas $n^{\circ}$ & $\begin{array}{l}\text { Origine } \\
\mathrm{P}: \text { producteur (natif) } \\
\mathrm{C}: \text { colon } \\
\mathrm{S}: \text { salarié }\end{array}$ & $\begin{array}{l}\mathrm{Nb} \text { main-d'œuvre } \\
\mathrm{T}: \text { temporaire } \\
\mathrm{P}: \text { permanente }\end{array}$ & $\begin{array}{l}\text { Diversification productive } \\
\text { Élevage mixte = bovins/ovins } \\
\text { QA = production de fromage }\end{array}$ \\
\hline $\begin{array}{l}\mathbf{1}=\text { Natifs } \\
\text { Éleveurs uruguayens } \\
\text { Nouvelle organisation }\end{array}$ & $\begin{array}{l}1 \\
2 \\
3 \\
4\end{array}$ & $\begin{array}{l}P \\
p \\
S+P \\
S+P\end{array}$ & $\begin{array}{l}\text { Oui }(1 \mathrm{~T}) \\
\text { Non } \\
\text { Non } \\
\text { Non }\end{array}$ & $\begin{array}{l}\text { Élevage mixte } \\
\text { Élevage mixte } \\
\text { Élevage mixte } \\
\text { Élevage mixte }\end{array}$ \\
\hline $\begin{array}{l}\mathbf{2}=\text { Natifs } \\
\text { Colons uruguayens } \\
\text { Organisation traditionnelle }\end{array}$ & $\begin{array}{l}5 \\
6 \\
7 \\
8\end{array}$ & $\begin{array}{l}\mathrm{C} \\
\mathrm{S}+\mathrm{C} \\
\mathrm{C} \\
\mathrm{S}+\mathrm{C}\end{array}$ & $\begin{array}{l}\text { Oui }(1 \mathrm{~T}) \\
\text { Non } \\
\text { Non } \\
\text { Non }\end{array}$ & $\begin{array}{l}\text { Élevage mixte } \\
\text { Élevage mixte } \\
\text { Élevage mixte } \\
\text { Élevage mixte (QA) }\end{array}$ \\
\hline $\begin{array}{l}\mathbf{4}=\mathbf{I m m i g r e ́ s} \\
\text { Colons russes } \\
\text { Organisation traditionnelle }\end{array}$ & $\begin{array}{l}13 \\
14 \\
15 \\
16\end{array}$ & $\begin{array}{l}\mathrm{S}+\mathrm{P}+\mathrm{C} \\
\mathrm{S}+\mathrm{C} \\
\mathrm{C} \\
\mathrm{C}\end{array}$ & $\begin{array}{l}\text { Non } \\
\text { Non } \\
\text { Non } \\
\text { Oui (2P) }\end{array}$ & $\begin{array}{l}\text { Lait (QA) } \\
\text { Lait } \\
\text { Lait } \\
\text { Lait }\end{array}$ \\
\hline
\end{tabular}

fonctionnement et les stratégies de résistance mises en évidence.

L'originalité de la méthode consiste à combiner données quantitatives et qualitatives. Elle s'appuie fortement sur la confiance établie avec l'agriculteur. Le « récit de vie» (histoire, trajectoire et décisions importantes; Dumez, 2011) permet de rendre compte de certaines dimensions de l'exploitation comme le cycle de vie, l'évolution des stratégies familiales et les relations famille/décisions des agriculteurs. Le récit de vie a été utilisé dans la première étape, principalement et de manière complémentaire à l'AGEA. Le postulat de cohérence de l'AGEA, «les producteurs ont des raisons de faire ce qu'ils font», renvoie à deux dimensions de l'habitus de Bourdieu (2001 : 241-242) : l'habitus est systématique (il y a concordance entre des pratiques agricoles différentes) et est transférable (d'une pratique à une autre, d'un domaine à un autre). 
Tableau 2. Méta-pratiques, domaines d'action et types de stratégies de résistance des agriculteurs familiaux.

Table 2. Types of resistance of family farmers according to the type of strategy.

\begin{tabular}{ll}
\hline $\begin{array}{l}\text { Méta-pratiques } \\
\text { (in vivo codes) }\end{array}$ & Domaines d'action \\
\hline $\begin{array}{l}\text { Travail propre } \\
\text { Travailler en famille } \\
\text { S'associer avec les voisins } \\
\text { Être de bons travailleurs (producteurs) } \\
\text { Communiquer }\end{array}$ & $\begin{array}{l}\text { Travail familial : travail familial autosuffisa } \\
\text { et avec partenariats de différentes modalités }\end{array}$ \\
$\begin{array}{l}\text { Minimiser l'appropriation des excédents (surplus) } \\
\text { Payer au comptant }\end{array}$ & $\begin{array}{l}\text { Commerce : établir des canaux de } \\
\text { commercialisation (contrats d'achat) qui } \\
\text { garantissent une autonomie, en évitant des } \\
\text { crédits et en diminuant les risques }\end{array}$ \\
\end{tabular}

Investir un peu et en attendre beaucoup (en élevage) Techniques-productives : se préparer afin de S'il y a des résultats, on ne change pas de méthodologie faire face aux crises (diversifier les produits Élevage mixte (ovins et bovins)

Assurer l'avenir, le bien-être de la famille Investir pour économiser

Grandir de l'intérieur de l'exploitation

Activités productives au compte des enfants

Bien faire les choses (le sens du travail).

Prioriser la famille.

Préserver une mode de vie

Donner une éducation aux enfants

Se faire connaître en tant que producteur (prestige) Accorder sa confiance et être digne de confiance
Type résistance

I Rendre la famille autonome

II Transmettre le patrimoine familial

Transmettre les valeurs nécessaires pour soutenir une «autonomie étique» (l'éthique paysanne)

Éducation scolaire : laisser un héritage du capital culturel à la génération suivante

Être reconnu par l'entourage

III Rester dans le réseau territorial
Pour pouvoir construire le modèle stratégique, l'AGEA propose un schéma conceptuel adapté aux conditions de l'agriculture familiale, dans la mesure où il concerne: i) les objectifs que la famille cherche à atteindre dans la vie et dans son travail ; ii) les règles qui délimitent le cadre de ses actions dans une situation donnée afin d'atteindre ses objectifs et iii) les actions ou décisions stratégiques. Il permet ainsi de «passer» des pratiques directement observables à la recherche, par un principe de cohérence, des régularités ou règles stratégiques qui permettent d'atteindre les motivations qui les précèdent (Landáis et al., 1988).

\section{Diversité des pratiques et stratégies de résistance}

Suivant la notion de système de reproduction proposée par Bourdieu (1994), nous avons identifié 70 pratiques de type économique, 20 de type symbolique, 19 de type éducatif et 10 de type successoral; soit un total de 115 pratiques classées selon leur nature ou leur visée, pour obtenir au total 22 métapratiques (Tab. 2). Nous les avons ensuite mises en relation avec les 8 domaines d'action qui correspondent à des « valeurs paysannes » et/ou des stratégies. En fonction de la complémentarité entre les domaines, nous les avons regroupées selon 3 types de résistance.

\subsection{Type I: «Rendre la famille autonome»}

Cette résistance est composée principalement de stratégies économiques. La stratégie de résistance économique, au sens de Bourdieu (1991), est la plus fréquente au sein des exploitations familiales. Son principal objectif est de maintenir l'autonomie de l'exploitation. Les études de cas ont permis d'identifier un ensemble de pratiques et de systèmes de règles stratégiques communes, matériellement et symboliquement liées, pour maintenir le travail familial au centre du système de l'exploitation.

Ici, l'existence d'un « habitus paysan » commun permet de proposer la présence d'un «sens du jeu» des producteurs familiaux uruguayens, qui s'intègre dans leurs actions pratiques et dans leur manière d'être et de percevoir le monde (nous appelons cela la «paysannerie »). Cela se traduit par des pratiques d'autocontrôle sur les ressources naturelles, de distanciation des marchés d'intrants, de commercialisation en circuit court et d'endettement minimal. 
Économie paysanne

Être un bon travailleur/producteur Réaliser des partenariats avec les voisins (métayage)

Développer les contrats informels, accords, arrangements («travailler de parole») Payer au comptant Minimiser l'extraction des excédents Diversifier l'élevage (ovin/bovin)

\section{Le métier d'agriculteur familial}

Être reconnu comme producteur (prestige) Obtenir la confiance des autres

Profiter des avantages de l'environnement (et de l'entourage)

Bénéficier des programmes de l'État

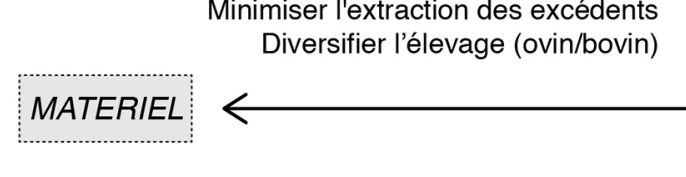

Modèle gestionnaire paysan

Privilégier le travail en famille Investir peu pour en attendre des fortes retombées Garder les techniques et les stratégies lorsqu'elles fonctionnent (les traditions également) Assurer l'avenir et le bien-être de la famille Croître de l'intérieur Gérer les activités productives tout en tenant compte des enfants

\section{INDIVIDUEL - FAMILIAL}

Fig. 2. Grille de lecture des méta-pratiques de résistance en fonction du niveau d'action et du registre de l'action.

Fig. 2. Reading grid of the rules and strategies of resistance based on the action levels and main dimensions of family farmers' practices.

\subsection{Type II: «Transmettre le patrimoine familial »}

Ce type de résistance est composé principalement des stratégies éducatives et successorales. La stratégie éducative la plus présente est l'éthique-ontologique, centrée sur l'économie familiale (Shanin, 2008) : "prioriser la famille», "préserver un mode de vie» où la famille joue un rôle central en fournissant la force de travail, les investissements... Les relations d'échanges avec d'autres agriculteurs n'impliquent pas nécessairement des transactions monétaires et la biodiversité représente une dimension importante des systèmes de production. La stratégie éducative formelle (système scolaire) tend à se généraliser.

\subsection{Type III : «Rester dans le réseau territorial »}

Ce type de résistance est le produit des stratégies et pratiques qui visent à maintenir la famille au sein des territoires et des réseaux socio-productifs. Elle a une composante symbolique importante: les stratégies sont en effet basées sur l'honneur («être une personne de confiance») et sur le prestige («faire les choses bien»). Elles prennent de l'importance au même titre que celles qui fabriquent du capital social collectif dans les réseaux d'échange. Elles sont au cœur de nombreuses stratégies économiques de résistance de type I. L'honorabilité et le prestige familial, par exemple, fonctionnent comme un capital symbolique fondamental dans le cas du métayage ou des arrangements productifs. Les échanges amicaux et la coopération entre voisins permettent, par exemple, à de nombreux jeunes d'avoir accès à des parcelles de pâture non exploitées pour initier leur activité d'élevage indépendamment de leur famille. Ce type de pratiques de résistance fabrique l'identité productive et rurale des territoires et facilite la transmission des exploitations familiales.

\section{Formes de résistance et habitus paysan}

Afin de comprendre comment les pratiques individuelles et collectives, constitutives de l'habitus, s'articulent pour donner naissance à des formes de résistance, nous avons élaboré une grille (Fig. 2) selon deux axes: un axe selon le domaine d'action des pratiques de résistance (individuel-familial ou collectif-professionnel), et l'autre selon le registre de l'action, matériel ou symbolique.

\section{1 Économie paysanne}

Les pratiques économiques, à la base de cette forme de résistance, structurent les relations typiques des éleveurs familiaux. La famille fonctionne comme un réseau primaire à partir duquel le travail collectif est organisé et le fonctionnement 
de la ferme est assuré. C'est une économie de type paysan, avec un niveau élevé de travail familial (« travailler au sein de la famille», «faire tout le travail») et avec une faible incidence de capital économique, peu ou pas de participation de la main-d'œuvre salariée ( «être autonome», «tout réaliser par son propre travail »), et des pratiques symboliques comme les associations de quartier («donner sa parole»), qui impliquent des relations de confiance et des valeurs partagées ("être de bons travailleurs»), toutes liées au capital social. Par ailleurs, les éleveurs développent des pratiques permettant d'éviter les crédits («manipuler de l'argent liquide»), un fonctionnement éloigné de la logique entrepreneuriale. Leur récurrence, dans nos cas, témoigne de la construction partagée à la fois d'une pratique qui permet d'assurer la pérennité de l'économie paysanne, et d'un mécanisme alternatif au modèle dominant. L'adoption de la technologie, ainsi que les programmes publics et privés qui imposent l'utilisation de crédits bancaires, tant pour les investissements que pour l'achat d'intrants, ont été l'une des causes historiques de l'endettement des producteurs agricoles et de leur faillite. L'économie paysanne est également basée sur des méta-pratiques techniques et productives qui visent à réduire les crédits bancaires. Nous illustrons ceci par les «paroles» de certains agriculteurs interrogés: «Non, avec l'aide de Dieu, je ne connais pas le crédit car l'idée est de se développer sans faire appel au crédit et d'investir seulement quand on peut»; " on se débrouille avec ce que nous avons dans l'exploitation, on utilise des techniques simples, élever les animaux nés dans l'exploitation de manière à investir peu et attendre beaucoup»; "se développer sans crédit et se développer seulement quand on peut, de manière à éviter les risques liés à l'endettement ».

\subsection{Le métier d’agriculteur familial}

Les méta-pratiques sont fondées sur la reconnaissance; elles mettent en jeu le prestige et l'honorabilité des familles. En l'absence d'autres capitaux, comme le capital économique, il est possible de mobiliser le capital social et symbolique sur la base de relations de confiance, ce qui caractérise la résistance de type III. Ainsi, certaines méta-pratiques visent à construire un «capital social familial» et se manifestent au travers de stratégies symboliques liées au maintien du prestige de la famille («participer aux activités collectives », «être référent » dans les activités collectives). Cela signifie que les autres agriculteurs peuvent compter sur eux. Il s'agit d'un capital social-réseau ayant des conséquences sur le capital économique. De plus, «être digne de confiance» fait référence à un ensemble de pratiques qui soutiennent, au sein de leurs réseaux, des échanges symboliques entre producteurs. Même sans capital économique, ils peuvent rendre viables leurs exploitations agricoles par le biais d'échanges, de travail d'entraide, « de bons procédés » ou/ et «d'échanges négociés» (troc d'animaux, de journées de travail, de machines, mais aussi de terres contre des journées de travail. Une pratique assez développée est la «capitalization» (qui consiste à échanger des pâtures contre des animaux nés lors de l'utilisation des pâtures). Un cas particulier de stratégie symbolique concerne la participation aux organisations syndicales : c'est le « capital militant ». Il permet aux éleveurs engagés de bénéficier plus facilement des informations et des programmes d'aide de l'État.

\section{3 Éthique/valeur de la famille paysanne}

Cette forme recouvre les méta-pratiques éducatives stratégiques, éthiques et scolaires. Les méta-pratiques éthiques structurent les modes de vie ainsi que le «producteur familial » lui-même. C'est un aspect fondamental de la reproduction sociale. Elles s'inscrivent, avec les méta-pratiques successorales, dans la forme de résistance «Métier». Cette éthique paysanne, au sens d'un système logique qui organise des valeurs, implique la transmission de valeurs familiales («prioriser la famille») et professionnelles («faire les choses bien ») différentes de celles promues par le modèle capitaliste de production basé sur le marché, l'individualisme ou la recherche du profit. Ainsi, tout en maintenant une «autonomie éthique», ces méta-pratiques récupèrent les actions stratégiques de l'habitus paysan, exprimées au sein de la «résistance » de l'économie paysanne face à l'économie de marché, et sont donc aussi à la base des résistances de type III. Cependant, elles visent toujours à accroître le capital culturel de la descendance et s'expriment dans deux sens différents et complémentaires : les enfants peuvent choisir (d'être agriculteur ou non); ils sont autonomes et indépendants (ils peuvent choisir de l'être à leur compte). Ces méta-pratiques varient selon les familles et les territoires, et constituent un exemple de la dynamique d'adaptation de l'habitus paysan aux changements d'environnement, en mobilisant les atouts et les différentes formes de capital propres à chacun.

\subsection{Modèle gestionnaire paysan}

Cette forme de résistance contient des méta-pratiques gestionnaires développées dans le système de petites exploitations : maximisation du travail familial (à la fois le « travail en famille» et de « tout faire par son propre travail »), réduction de la dépendance au capital économique («investir un peu pour en attendre beaucoup» ou «investir pour économiser») et limiter les risques aux niveaux technique et productif («si ça marche, on ne change rien»). Avec les autres pratiques exprimées dans l'«Économie paysanne», elles sont à la base de la résistance de type I. Il est à noter que les stratégies économiques fonctionnent comme un système stratégique unique, renforcé par son imbrication forte dans d'autres stratégies non économiques (comme les stratégies successorales, d'investissement éducatif ou symboliques). Elles sont donc aussi à la base de la résistance de type II, puisque la transmission du patrimoine familial implique un certain «bien-être familial», permet d' «assurer le futur» et d'assurer la succession en augmentant le patrimoine («grandir de l'intérieur»), plutôt que par le développement des «activités productives à la charge des enfants ».

En résumé, ces méta-pratiques de résistance se réfèrent à certaines «règles» dans l'action des producteurs familiaux. Elles produisent du sens pratique qui structure l'habitus paysan et reproduit une autonomie éthique propre à ces producteurs. L'habitus paysan permet aux producteurs de maintenir le caractère familial de leur activité et sert de "guide» aux stratégies de résistance pour faire face aux modèles dominants. L'analyse de ces stratégies montre comment elles permettent de stabiliser les valeurs des exploitations familiales tout en les diversifiant et en les adaptant. 


\section{Conclusion}

L'objectif de cet article était double : d'une part, contribuer à améliorer les connaissances sur les formes et les mécanismes développés par les éleveurs familiaux uruguayens pour résister au modèle de production dominant qui privilégie l'intensification et les bénéfices immédiats au détriment de la protection des ressources naturelles et des conditions de vie des agriculteurs familiaux. D'autre part, il s'agissait de tester la pertinence de la notion d'habitus afin de comprendre le poids des valeurs et de l'éthique paysanne dans la fabrique du métier d'agriculteur. Les résultats sont encourageants et montrent que la notion d'habitus est un concept analytique qui oblige à considérer de manière simultanée la dimension «matérielle » et la dimension «symbolique». Cependant, comme le signalent divers travaux, la dimension collective joue un rôle important dans la fabrique du métier d'agriculteur. Cette dimension devrait être davantage explorée dans les travaux futurs en Uruguay en particulier concernant l'agroécologie. En effet, la mise en place de l'agro-écologie est une bonne opportunité pour étudier les rôles des pratiques individuelles et collectives dans la fabrique de l'agro-écologie.

Malgré l'importance des stratégies d'investissement symbolique dans l'activité des producteurs, les chercheurs et les décideurs politiques leur accordent relativement peu d'attention. Par ailleurs, nous notons que ces stratégies ne suffisent pas à assurer la pérennité de la production familiale et les efforts des politiques ciblées semblent jusqu'à présent agir lentement et n'arrivent pas à contrecarrer les tendances qui menacent leur reproduction sociale. Cependant, le nouveau projet agro-écologique pour l'Uruguay peut inverser cette tendance, car sa cible principale est l'agriculture familiale et en particulier les éleveurs qui représentent la majorité des exploitants. La concentration, l'internationalisation des terres, ainsi que l'expansion du modèle de production dominant, constituent la principale menace à la persistance de la production familiale dans le milieu rural et à la durabilité des campagnes uruguayennes.

L'analyse des cas a permis d'identifier des ensembles de pratiques et des systèmes de règles stratégiques communes, qui sont matériellement et symboliquement liés au maintien du travail familial dans le «système famille-exploitation», bien qu'elles évoluent au cours du temps. Les agriculteurs familiaux uruguayens ont un certain "sens du jeu», agissant sur leurs actions pratiques et sur leur manière d'être et de percevoir le monde : c'est « la paysannerie».

Conflits d'intérêt. Les auteurs déclarent n'avoir aucun lien d'intérêt concernant les données publiées dans cet article.

\section{Financements}

Cette recherche n'a reçu aucune subvention spécifique d'agences de financement des secteurs public, commercial ou à but non lucratif.

\section{Références}

Achkar M, Domínguez A, Pesce F. 2006. Principales transformaciones territoriales en el Uruguay rural contemporáneo. Pampa 2: 219-242.
Bourdieu P. 1991. El sentido práctico. Madrid: Taurus, 451 p.

Bourdieu P. 1994. Stratégies de reproduction et modes de domination. Actes de la recherche en sciences sociales 105: 3-12.

Bourdieu P. 2001. Las estructuras sociales de la economía. Buenos Aires: Manantial, $270 \mathrm{p}$.

Bourdieu P, Wacquant L. 2008. Una invitación a la sociología reflexiva. Buenos Aires: Siglo XXI, 412 p.

Carámbula M. 2015. Imágenes del campo uruguayo en-clave de metamorfosis: Cuando las bases estructurales se terminan quebrando. Revista de Ciencias Sociales 28(36): 17-36.

Chia E. 1990. L'organisation de l'économie paysanne. Économie rurale 200: $85-86$.

Chia E, Dugué P (coordinateurs). 2006. L'exploitation agricole familiale : mythe ou réalité ? Cahiers Agricultures 15(6): 483-604.

De Certeau M. 1980. L'invention du quotidien. Paris : UGE. Dumez H. 2011. Qu'est-ce que la recherche qualitative? Le Libellio d'AEGIS 7(4): 47-58.

Dumez H. 2011. Qu'est-ce que la recherche qualitative? Le Libellio d'AEGIS 7(4): 47-58.

Gasselin P, Choisis JP, Petit S, Purseigle F, Zasser S (eds). 2014. Ĺagriculture en famille : travailler, réinventer, transmettre. France : INRA-SAD, $382 \mathrm{p}$.

Landáis É, Deffontaines JP, Benoit M. 1988. Les pratiques des agriculteurs : point de vue sur un courant nouveau de la recherche agronomique. Études rurales 109: 125-158. DOI: 10.3406/ rural.1988.3226.

Latour B. 1993. Nous n'avons jamais été modernes: essai d'anthropologie symétrique. Paris: La Découverte, 213 p.

Levi-Strauss C. 1962. La pensée sauvage. Paris: Plon.

Marshall, E, Bonneviale JR, Francfort, I. 1994. Fonctionnement et diagnostic global de lexploitation agricole. Une méthode interdisciplinaire pour la formation et le développement. Dijon (France) : ENESAD-SED, $173 \mathrm{p}$.

Ministerio de Ganadería, Agricultura y Pesca (MGAP). 2014. Censo General Agropecuario, 2011, resultados definitivos. Montevideo: MGAP, 142 p. Disponible sur https://www.gub.uy/ministerioganaderia-agricultura-pesca/datos-y-estadisticas/estadisticas/ censo-general-agropecuario-2011\#dropdown.

Ministerio de Ganadería, Agricultura y Pesca (MGAP). 2018. Anuario estadístico agropecuario 2018. Montevideo: MGAP, 210 p. Disponible sur http://www.mgap.gub.uy/unidad-organiza tiva/oficina-de-programacion-y-politica-agropecuaria/estadisticasy-documentos/29-08.

Osty PL. 1978. L'exploitation agricole vue comme un système: diffusion de linnovation et contribution au développement. Bulletin technique dinformations (BTI) 326: 43-49.

Oyhantçabal G, Narbondo I. 2019. Land grabbing in Uruguay: New forms of land concentration. Canadian Journal of Development Studies 40(2): 201-219. DOI: 10.1080/02255189.2018.1524749.

Piñeiro D. 1985. Formas de resistencia de la agricultura familiar: El caso del noreste de Canelones. Montevideo: CIESU, 177 p. (Estudios sobre la sociedad uruguaya, 6).

Piñeiro D. 2014. Asalto a la tierra: El capital financiero descubre el campo uruguayo. In: Almeyra G, Concheiro Bórquez L, Mendes Pereira JM, Porto-Gonçalves CW. Capitalismo: Tierra y poder en América Latina (1982-2012) Argentina, Brasil, Chile, Paraguay, Uruguay. Buenos Aires: Continente, pp. 215-257.

Ploeg JD van der. 2010. The peasantries of the twenty-first century: The commoditisation debate revisited. The Journal of Peasant Studies 37(1): 1-30. DOI: 10.1080/03066150903498721.

Reed-Danahay D. 2007. De la résistance: éthnographie et théorie dans la France rurale. Éducation et sociétés 19: 115-131.

Rossi V. 2017. Prácticas de resistencia de la producción familiar en el agro uruguayo. (Tesis de doctorado). Córdoba: Universidad 
Nacional de Córdoba, 375 p. Disponible sur https://rdu.unc.edu.ar/ handle/11086/14600.

Schneider S. 2016. Family farming in Latin America and the Caribbean: Looking for new paths of rural development and food security. Brasília: FAO, 42 p. (International Policy Centre for Inclusive Growth (IPC-IG), Working Paper, No. 137).
Scott JC. 1989. Everyday forms of resistance. The Copenhagen Journal of Asian Studies 4: 33-62. DOI: 10.22439/cjas.v4i1.1765. Shanin T. 2008. Lecciones campesinas. In: Paulino ET, Fabrini JE, org. Campesinato e territórios em disputa. São Paulo: Expressão Popular, $495 \mathrm{p}$.

Sourisseau JM (ed). 2014. Agricultures familiales et mondes à venir. Paris: Quae, 360 p.

Citation de l'article : Rossi V, Filardo V, Chia E. 2020. Stratégies de résistance des agriculteurs familiaux face à l'avancée de l'agriculture capitaliste en Uruguay. Cah. Agric. 29: 16. 